\title{
Guest editorial: special issue on data and artifact-centric business processes
}

\author{
Marlon Dumas $^{1}$ (D) Richard Hull ${ }^{2} \cdot$ Fabio Patrizi $^{3}$
}

Published online: 9 February 2016

(C) Springer-Verlag Wien 2016

Traditionally, researchers in the field of Business Process Management (BPM) have focused on studying control-flow aspects of business processes independently from data aspects. The separation of concerns between control-flow and data has been fruitful and has enabled the development of various foundational theories and methods for BPM. However, the limits of theories and methods built on this separation of concerns have now become evident, particularly with the increasing pressure to support ad hoc and flexible business processes, where control-flow is often intermingled with data.

In the past decade, various approaches have emerged that emphasize the integration of data and control as key pillars to support the specification, analysis, enactment, and monitoring of rich and flexible business processes. These include object-centric and artifact-centric approaches, where data and behavior are bundled together into logical units, as well as case management approaches, where a process is viewed as cases where data and documents circulate across multiple actors in a way that is not necessarily driven by normative procedures. This special issue focuses on the former family approaches, where research has attained a certain level of maturity in recent years.

The issue showcases four research contributions pertaining to data- and artifactcentric approaches to BPM.

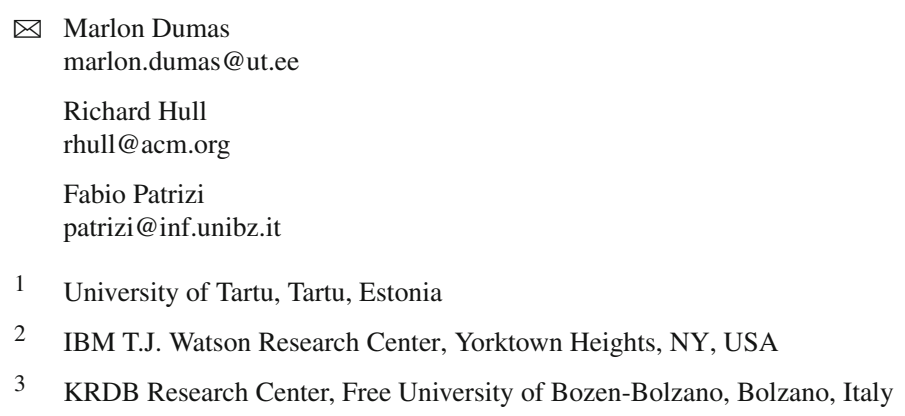


The first contribution ("Synthesizing Data-Centric Models from Business Process Models" by Eshuis \& Van Gorp) is representative of a line of research that bridges across imperative control flow-oriented paradigms for process modeling, and declarative data-centric ones. The paper proposes a transformation from a process model captured as a UML Activity Diagram to a set of state machines describing the lifecycle of object types involved in the process. These state machines are then transformed into Guard-Stage-Milestone (GSM) specifications. GSM is a language designed to specify artifact-centric processes declaratively and in a way that data and control-flow are captured in an integrated manner.

In the second contribution ("A data-centric approach to manage business processes"), Haddar et al. put forward a new meta-model for capturing control-flow and data in an integrated manner. The authors take a popular formalism for modeling business processes, namely Petri nets, and extend it by mapping the places in the net to relational entities that hold structured tokens (data tuples), and mapping the transitions in the net to data operations inspired from relational algebra. The proposed meta-model is embodied in a workflow management system, namely the Opus Workflow System, which supports the definition and execution of processes captured in the proposed meta-model, as well as the specification of schema matchings necessary to map data across relational entities. The article also outlines techniques for model verification, including deadlock detection.

The third contribution ("Balanced Multi-Perspective Checking of Process Conformance" by Mannhardt et al.) addresses the question of integrating the control-flow and data perspectives when checking the conformance of event logs against process models. This article is representative of a body of research that seeks to extend business process mining techniques - traditionally focused on control-flow aspects-in order to take data into account. As in the previous contribution, Petri nets extended with data elements (so-called DPN-nets) are used to capture process models. The article presents a notion of alignment between an event log consisting of business process execution traces and a DPN-net, as well as an algorithm to compute an optimal alignment.

Finally, in the fourth contribution ("Enabling coupled multi-scale, multi-field experiments through choreographies of data-driven scientific simulations") Weiss \& Karastoyanova focus on a well-known application domain of process management, namely scientific workflows, which are known for their data-driven nature. Specifically, the article presents an approach to model multi-scale and/or multi-field scientific simulations using the concept of choreography, wherein a collaborative process is captured as a set of independent processes or services that collectively interact to achieve a given goal. The proposed approach demonstrates how concepts developed in the field of control flow-oriented process modeling can be transposed to application domains where processes are mostly data-driven.

Collectively, the four contributions are representative of ongoing research towards the elusive quest of developing process modeling, enactment and monitoring methods and tools that seamlessly integrate control-flow and data and hence allow for a more holistic verification, analysis and monitoring of business processes. We hope that this special issue will foster further research in the field and raise interest from other research communities. 